\title{
An effective elastic thickness map of Australia from wavelet transforms of gravity and topography using Forsyth's method
}

\author{
C. J. Swain ${ }^{1,2}$ and J. F. Kirby ${ }^{1}$ \\ Received 30 October 2005; revised 14 December 2005; accepted 20 December 2005; published 27 January 2006.
}

[1] We have developed a wavelet version of Forsyth's coherence method for estimating the effective elastic thickness $\left(T_{e}\right)$ of the lithosphere from gravity and topography. We have previously shown that the 'fan' wavelet is particularly well suited to making localised coherence estimates and here we progress the method by showing how the coherence can be modelled by using the wavelet transforms of gravity and topography to infer the initial loads that flex the plate. The effectiveness of the method is demonstrated on synthetic data from a model having a rectangular area of large $T_{e}$ embedded in a uniform low $T_{e}$ surrounding, and a load ratio that increases with wave number. The results for the Australia region show a 'core' of high $T_{e}$ under north and central Australia, with much lower values to the east and also, unexpectedly, under the western parts of the Yilgarn and Pilbara cratons. Citation: Swain, C. J., and J. F. Kirby (2006), An effective elastic thickness map of Australia from wavelet transforms of gravity and topography using Forsyth's method, Geophys. Res. Lett., 33, L02314, doi:10.1029/2005GL025090.

\section{Introduction}

[2] The coherence method [Forsyth, 1985] has been widely used for estimating effective elastic thickness $\left(T_{e}\right)$ of continental regions. Originally the method used periodogram spectral estimates, often on data sets that had been mirrored in their edges [e.g., Zuber et al., 1989]. More recently the method has been enhanced to allow mapping $T_{e}$ by making maximum entropy or multitaper spectral estimates in a regular array of windows [Lowry and Smith, 1995; Pérez-Gussinyé and Watts, 2005]. The model used in all these studies is a uniform elastic plate, which flexes under both surface (topographic) and subsurface loads. In Forsyth's [1985] method these initial loads are estimated from the observed topography and gravity, assuming a value for $T_{e}$, which allows a predicted coherence to be calculated. $T_{e}$ can then be estimated as the value that minimizes the misfit between observed and predicted coherence. By working in the wave number domain, where, for a uniform plate, the differential equation for plate bending becomes a linear equation, estimation of the initial loads is reduced to solving 2 simultaneous equations at each 2-D wave number [Forsyth, 1985].

[3] All the studies referred to use the windowed Fourier transform, creating the obvious dilemma [Kirby and Swain,

\footnotetext{
${ }^{1}$ Western Australian Centre for Geodesy, Curtin University, Perth, Western Australia, Australia.

${ }^{2}$ Permanently at Booragoon, Western Australia, Australia.
}

2004] that the window must be large enough to measure the transition from high to low coherence, while use of a large window will severely restrict the resolution possible. For example, Pérez-Gussinyé and Watts [2005] note that for a window size of $1000 \times 1000 \mathrm{~km}$ the largest $T_{e}$ value they can reliably recover is $60 \mathrm{~km}$; their final map is based on a number of different window sizes. The use of wavelets solves this dilemma in a natural way, essentially using an optimal sized window for each wave number, or 'scale'. Stark et al. [2003] were the first to apply a 2-D wavelet transform to map $T_{e}$ variations. This transform allows a local wavelet coherence (and admittance) to be calculated as a function of scale at each point of a data grid. They did not use Forsyth's method to estimate the initial loads, but instead estimated $T_{e}$ and the load ratio $f$ at each point from the coherence and the 'pseudo-coherence' - a composite of the observed admittance and the predicted coherence and admittance. This 'normalisation' of the admittance was found necessary because the raw wavelet admittance is very noisy. Kirby and Swain [2004] introduced the 'fan' wavelet for estimating the coherence, finding it superior to the wavelets used by Stark et al. [2003] because estimates for synthetic data agree very well with theoretical curves, provided the 'equivalent Fourier wave number' is used. This simplifies the process of inversion considerably. Kirby and Swain [2004] limited their application of the method to recovery of $T_{e}$ for a simple synthetic model with varying $T_{e}$, assuming $f=1$. We have tried a number of approaches to the simultaneous estimation of $T_{e}$ and $f$ at each point, including using the 'pseudo-coherence', and have found that the latter can yield excellent results with synthetic data. With real data, however we frequently find that the variations of the admittance are so large that the method is unstable. Here we show that Forsyth's method is easily adapted for use with the fan wavelet, allowing the calculation, at each grid point, of initial surface and subsurface loads as functions of scale, and that the method is robust and works with both synthetic and real data.

\section{Forsyth's Method Using the Fan Wavelet Transform}

[4] As noted above, Forsyth's method estimates the initial loads from gravity and topography data that are functions of wave number. In the conventional implementation the latter are simply the Fourier transforms of the data; the initial loads are then used to calculate the four components of surface and subsurface topography that they cause. Predicted coherence as a function of 1-D wave number is then found by averaging the auto- and crossspectra of the four components between wave number annuli as by Forsyth [1985, equation (25)]. Note that this 


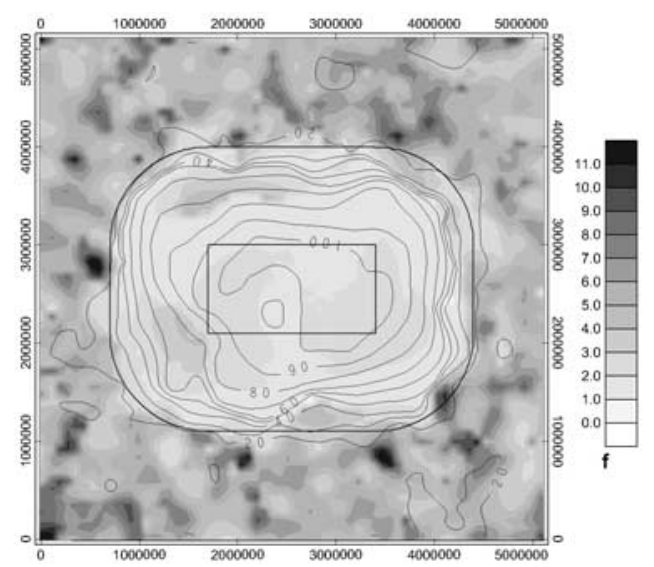

Figure 1. Results for the 'bump' model of applying Forsyth's method at each point. Contours show $T_{e}$ distribution $(\mathrm{km})$. Grey scale image is average load ratio $f$ in the transition waveband. Contours of the maximum $(150 \mathrm{~km})$ and minimum $(20 \mathrm{~km})$ model $T_{e}$ values are also shown.

is equivalent to calculating $f(k)$ from the ratio of subsurface to surface load [Banks et al., 2001, equation (10)] and then inserting this into the equation for theoretical coherence [e.g., Kirby and Swain, 2006, equation (A5)].

[5] Here we use the wavelet transforms of the gravity and topography data, with their scales converted to equivalent Fourier wave numbers [Kirby and Swain, 2004]. Forsyth's [1985] equations (13)-(21) are applied to these two wavelet transforms (at each point) to calculate the four components of surface and subsurface topography. Then advantage is taken of the fact that the fan wavelet consists of the sum of a number ( $N_{\theta}$, usually 11 for the isotropic case) of component wavelets at a range of azimuths so that the wavelet auto- and cross-spectra can be averaged and used to calculate the predicted coherence.

[6] Note that the model suggested by Forsyth [1985] assumes initial loading by relief at the Moho whose estimation involves downward continuation of Bouguer gravity. This may generate numerical instabilities where the Moho is particularly deep, if the anomalies actually have shallower sources. This can be avoided by using an alternative loading model of density variations in a thin layer at an appropriate depth within the crust [Banks et al., 2001; Swain and Kirby, 2003a]. However, we have not found it to be a problem with the Australian data so, although we have developed wavelet versions for both models, for simplicity we restrict this study to the use of Forsyth's model. Then the only parameter we need to assume, other than densities, is crustal thickness. A second potential problem is that the solution for the loads can be nearly singular at long wavelengths [Forsyth, 1985]. Fortunately this does not seem to be a problem with wavelets: in all the cases we have seen, the loading ratio decreases smoothly to low values at the longest wavelengths, as expected. This is a significant advantage and may be because we are solving the load equations using as data the wavelet transforms, which are smooth and overlap each other in the wave number domain, instead of raw Fourier coefficients.

[7] An underlying assumption here is that local wavelet spectra are independent or 'decoupled' from the spectra at adjacent points [Stark et al., 2003], i.e., that the coherence spectrum at a point is only affected by the plate thickness and loading directly below that point. We shall note some limitations of this approximation in the next section.

\section{Synthetic Model Results}

[8] We have implemented the method in 3 stages. First we computed the wavelet transforms of the gravity and topography grids at each scale and each azimuth of the fan wavelet [Kirby and Swain, 2004]. From these transforms, we calculated the wavelet auto- and cross-spectra and hence the coherences [Kirby and Swain, 2004, equation (5)] and their uncertainties. For the latter we here used the jackknife method [Thomson and Chave, 1991] which derives statistics from pseudo-values formed by deleting single points from the spectral averages. For the coherence, we sometimes additionally average the spectra over blocks of $2 \times 2$ or more grid points. However, this hardly affects the results, which are effectively smoothed by the filtering inherent in the wavelet transform; moreover the jackknife statistics are calculated only from the $N_{\theta}$ different transforms. Finally we apply Brent's method of 1-D minimisation [Press et al., 1992] to find the $T_{e}$ that gives the best-fitting predicted coherence calculated from the wavelet transforms using Forsyth's method, where a misfit is weighted by the inverse of the uncertainty in an observed coherence. The loading ratio $f(k)$ is a by-product and can be averaged over the transition wave numbers to give a single value at each grid point.

[9] We have chosen a model having the same general 'geometry' as the 'bump' model by Kirby and Swain [2004], but with $T_{e}$ and $f$ distributions giving results that are similar in some ways to our results for Australia. A 'flat' rectangle in the middle with $T_{e}=150 \mathrm{~km}$ is surrounded by a linear 'ramp' $1000 \mathrm{~km}$ wide down to a uniform $T_{e}=20 \mathrm{~km}$ peripheral area (Figure 1). Grids, measuring $256 \times 256 \times 20 \mathrm{~km}$, of gravity and topography data were synthesized for this $T_{e}$ model, as described by Kirby and Swain [2004]. The loading ratio $f$ was made to increase with wave number to simulate the Australian data, as by Swain and Kirby [2003a].

[10] 'Slice' plots of the 3D coherence distribution are a good way to illustrate the limitations of the assumption of independence of local wavelet spectra. For example, Kirby and Swain [2004, Figure 4] shows a 'high coherence overhang' toward the east end of the mid-section, where $T_{e}$ is changing, showing the influence of adjacent lower $T_{e}$ values. Similar features can be seen in many $3 \mathrm{D}$ coherence plots for both real and synthetic data.

[11] Figure 2 gives the results of applying Forsyth's method at a point where the model $T_{e}$ is $20 \mathrm{~km}$. It shows the observed and best-fitting predicted coherences and admittances. Results tend to be smoother, and errors lower, over the areas of lower $T_{e}$ than over the high $T_{e}$ 'bump', but these plots are not exceptional. It can be seen that the admittance is implicitly fitted by the load calculation [Forsyth, 1985]. Figure 2 also shows the loading ratio $f$ which can be seen to increase from $<1$ at the longest wavelength to $>10$ at wavelengths $<100 \mathrm{~km}$, in accordance with the model [Swain and Kirby, 2003a].

[12] The result of applying Forsyth's method at all points is shown in Figure 1, which also shows top and bottom 

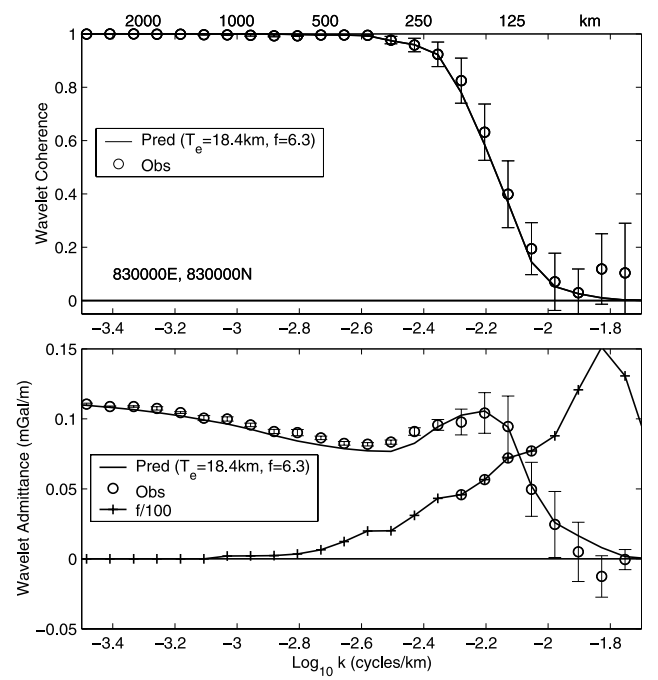

Figure 2. Results of applying Forsyth's method to wavelet coherence and wavelet transforms of Bouguer gravity and topography for the 'bump' model, at point (830000, 830000). (top) Observed wavelet coherence and best-fitting predicted coherence. (bottom) Observed and predicted wavelet admittance. The loading ratio is also shown, as $f / 100$, on the same scale (i.e., the $f$ scale goes from 0 to 15 ). The 4 values used to get the average $f$ are marked with circles.

contours of the model. It is worth noting that this is just one example from an infinite set of models that could be generated from fractal grids of data for this $T_{e}$ model. The 'base contour' of the model is well reproduced in this case, but elsewhere in the region of changing $T_{e}$ the fact that the coherence spectra are evidently influenced by adjacent $T_{e}$ values implies that the $T_{e}$ estimates themselves will be affected. For example, the maximum amplitude of the $T_{e}$ anomaly is less than the model maximum $(150 \mathrm{~km})$ simply because of its small width (less than the flexural wavelength of $1265 \mathrm{~km})$. Variations in the transition values of $f$ (grey-scale image in Figure 1) correspond roughly with the increase in $f$ with wave number inherent in the model; there are evidently large variations in the output $f$ in the low $T_{e}$ region, but these do not seem to correlate with the $T_{e}$ variations in this region.

[13] We have also applied this method to a similar model in which the 'ramp' was only $100 \mathrm{~km}$ wide, giving a much sharper $T_{e}$ contrast. The resulting $T_{e}$ map was similar to Figure 1, except that the low value contours $(20-60 \mathrm{~km})$ were much closer together, defining an 'edge' lying somewhat outside the outline of the true $T_{e}$ contrast. It appears that the method can distinguish between sharp and gradual $T_{e}$ variations, at least with large contrasts. But spurious anomalies sometimes occur, and can be larger than those in Figure 1.

\section{Results for Australia}

[14] Grids of complete Bouguer gravity and topography/ bathymetry data for Australia were prepared using a $20 \mathrm{~km}$ point spacing and an equatorial Mercator projection using data from Geoscience Australia, the GEBCO Digital Atlas and the KMS02 satellite altimetry model of Andersen and
Knudsen [1998]. Bathymetry was converted to equivalent rock load as described by Stark et al [2003]; that is, we scaled the depths by a factor $\left(\rho_{\mathrm{c}}-\rho_{\mathrm{w}}\right) / \rho_{\mathrm{c}}$, where $\rho_{\mathrm{c}}, \rho_{\mathrm{w}}$ are densities of the crust and sea water. The bathymetry signal is dominant in our data at the largest wavelet scales so, without this conversion, wavelet admittance is $\sim-0.07 \mathrm{mGal} / \mathrm{m}$ at these scales everywhere, even in the centre of Australia where it should be $\sim-0.11$. This remains a problem if, at sea, we use the corrected load equations which allow for the fluid/rock interface. The compromise we have used is to first convert the bathymetry as described above, then, before applying these modified load equations, rescale the wavelet transform of topography for points at sea by the inverse of the above factor.

[15] We applied Forsyth's method to these grids as described in Section 3, except that (a) we used CRUST 2.0 (http://mahi.ucsd.edu/Gabi/rem.html) for the crustal thickness at each point, and (b) we did not weight the coherence misfits as the inverse of their uncertainty estimates. Instead we used an inverse wave number weight function normalised to a value of 0.6 at the highest wave number. We found that this produces smoother results as it downweights the irregular high coherences that sometimes occur at high wave number. Note that synthetic data sets appear to be less sensitive to the type of weighting used. Apart from this problem, plots of 1-D coherence appear similar in quality to those from synthetic data. However, 1-D admittance plots can be highly irregular, a point noted by Stark et al. [2003]. Despite this, we usually find that at transition wave numbers the method fits them with the predicted admittances, giving stable, smoothly varying results.

[16] Figure 3 shows the variations of wavelet $T_{e}$ over Australia as contours, with the average $f$ at transition wave numbers being shown as a grey-scale image. Bearing in mind our results from synthetic models we give the following tentative description of the $T_{e}$ variation over Australia. The highest $T_{e}$ values are confined to central Australia, though relatively high values occur in northern Australia, including the Kimberley, and extend over the continental shelf to the north. The transition from

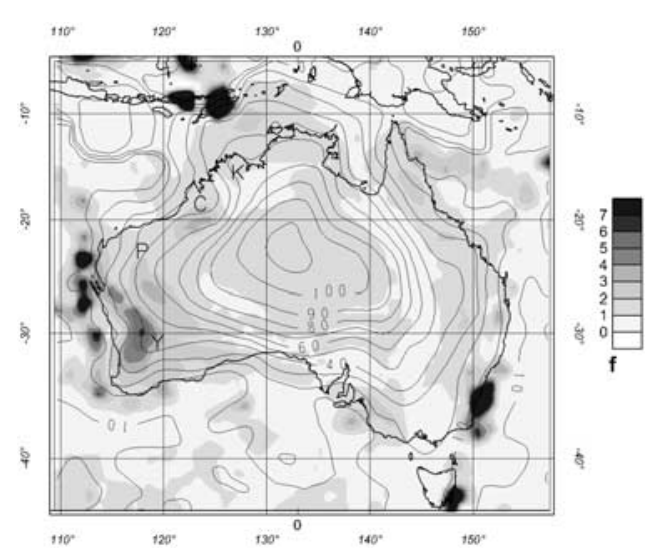

Figure 3. Results for Australia from applying Forsyth's method at each point. Contours show $T_{e}$ distribution $(\mathrm{km})$. Grey scale image is average load ratio in the transition waveband. $\mathrm{Y}=$ Yilgarn Craton; $\mathrm{P}=$ Pilbara Craton; $\mathrm{C}=$ Canning Basin; $\mathrm{K}=$ Kimberley Craton. 
high to low $T_{e}$ is fairly gradual over all of eastern Australia and much of Western Australia, somewhat sharper over the southern half of South Australia and also the NE part of Western Australia, where values drop very rapidly to a relative low over the Canning Basin. Values appear to be relatively low $(<60 \mathrm{~km})$ over the Pilbara and SW half of the Yilgarn craton. Interestingly, the Yilgarn is the only substantial land area with $f$ greater than about 3 . If $f$ were smaller here, $T_{e}$ would also be smaller. Offshore $T_{e}$ values are mainly $5-15 \mathrm{~km}$, though we are not inclined to rely too much on these results because the GEBCO bathymetry depends entirely on ship tracks which are very sparse in places.

\section{Discussion and Conclusions}

[17] Swain and Kirby [2003a, 2003b] gave corrected multitaper $T_{e}$ estimates for central Australia of $115 \pm$ $25 \mathrm{~km}$ and $100 \pm 20 \mathrm{~km}$, which agree with Figure 3. Zuber et al. [1989] used the 'standard' periodogram method, with mirroring, to make $T_{e}$ estimates in 12 rectangular windows over the continent. We have compared these to values at the centres of their windows superimposed on our map and expressed the result as a set of percent differences (i.e., $100 \times$ difference/mean). The mean difference is $7 \%$, but this disguises the fact that their 8 results for eastern Australia are on average $9 \%$ lower than ours, while the 4 results from the central and western parts average $40 \%$ higher. The largest difference (82\%) occurs for their 'WS' data set, a $1100 \times 1500 \mathrm{~km}$ rectangle covering the Yilgarn and Pilbara cratons, for which their estimate is $132 \mathrm{~km}$. There are several possible explanations for these large differences. (a) Macario et al. [1995] showed by means of synthetic data that the standard method could overestimate $T_{e}$ when the window is not much larger than the flexural wavelength, as is the case here. Swain and Kirby [2003b] note that this may be a result of the annular averaging used in this method. (b) Mirroring the data can introduce artefacts into the spectral estimates at long wavelengths causing significant, but unpredictable, changes in $T_{e}$ estimates. (c) There may be some edge-effect, due to the proximity of the cratons to the coast, causing a downward bias on our wavelet $T_{e}$ estimates, but this is speculative as we have not been able to simulate it with our synthetic models.

[18] As a further check we made multitaper $T_{e}$ estimates in three $1100 \times 1500 \mathrm{~km}$ windows centred on the WS, NCNO (Northern Craton) and CBB (Central Blocks) windows of Zuber et al. [1989]. We used the same sized window for each one because the downward bias caused by multitapers depends on window size [Swain and Kirby, 2003a]. Our 3 estimates were 55, 60 and $70 \mathrm{~km}$, where those of Zuber et al. [1989] are 132, 134 and $88 \mathrm{~km}$, respectively. The approximate wavelet $T_{e}$ estimates at the centres of these windows are 55, 80 and $100 \mathrm{~km}$, which, allowing for the downward bias, which increases nonlinearly with the true $T_{e}$ [Swain and Kirby, 2003a], agree better with the multitaper estimates than with those of Zuber et al. [1989]. Thus we consider the above explanations (a) or (b) to be more likely than (c). Nevertheless the wavelet $T_{e}$ values over the western parts of the Yilgarn and Pilbara cratons are low compared to most other cratons worldwide [e.g., Watts, 2001].

[19] Acknowledgment. We thank Geoscience Australia for the supply of terrestrial gravity and topography data, and Ole B. Andersen and Per Knudsen for the altimetry gravity grid.

\section{References}

Andersen, O. B., and P. Knudsen (1998), Global marine gravity field from the ERS-1 and Geosat geodetic mission altimetry, J. Geophys. Res., 103, $8129-8137$

Banks, R. J., S. C. Francis, and R. G. Hipkin (2001), Effects of loads in the upper crust on estimates of the elastic thickness of the lithosphere, Geophys. J. Int., 145, 291-299.

Forsyth, D. W. (1985), Subsurface loading and estimates of the flexural rigidity of continental lithosphere, J. Geophys. Res., 90, 12,623-12,632.

Kirby, J. F., and C. J. Swain (2004), Global and local isostatic coherence from the wavelet transform, Geophys. Res. Lett., 31(24), L24608, doi:10.1029/2004GL021569.

Kirby, J. F., and C. J. Swain (2006), Mapping the mechanical anisotropy of the lithosphere using a 2D wavelet coherence, and its application to Australia, Phys. Earth Planet. Inter., in press.

Lowry, A. R., and R. B. Smith (1995), Strength and rheology of the western U.S. Cordillera, J. Geophys. Res., 100, 17,947-17,963.

Macario, A., A. Malinverno, and W. F. Haxby (1995), On the robustness of elastic thickness estimates obtained using the coherence method, J. Geophys. Res., 100, 15,163-15,172.

Pérez-Gussinyé, M., and A. B. Watts (2005), The long-term strength of Europe and its implications for plate-forming processes, Nature, 436, 381-384, doi:10.1038/nature03854.

Press, W. H., S. A. Teukolsky, W. T. Vetterling, and B. P. Flannery (1992), Numerical Recipes in Fortran, Cambridge Univ. Press, New York.

Stark, C. P., J. Stewart, and C. J. Ebinger (2003), Wavelet transform mapping of effective elastic thickness and plate loading: Validation using synthetic data and application to the study of southern African tectonics, J. Geophys. Res., 108(B12), 2558, doi:10.1029/2001JB000609.

Swain, C. J., and J. Kirby (2003a), The effect of 'noise' on estimates of the elastic thickness of the continental lithosphere by the coherence method, Geophys. Res. Lett., 30(11), 1574, doi:10.1029/2003GL017070.

Swain, C. J., and J. Kirby (2003b), The coherence method using a thin anisotropic elastic plate model, Geophys. Res. Lett., 30(19), 2014, doi:10.1029/2003GL018350.

Thomson, D. J., and A. D. Chave (1991), Jackknifed error estimates for spectra, coherences, and transfer functions, in Advances in Spectrum Analysis and Array Processing, edited by S. Haykin, vol. 1, chap. 2, pp. 58-113, Prentice Hall, Englewood Cliffs, N. J.

Watts, A. B. (2001), Isostasy and Flexure of the Lithosphere, Cambridge Univ. Press, New York.

Zuber, M. T., T. D. Bechtel, and D. W. Forsyth (1989), Effective elastic thickness of the lithosphere and the mechanisms of isostatic compensation in Australia, J. Geophys. Res., 94, 9353-9366.

J. F. Kirby, Western Australian Centre for Geodesy, Curtin University, GPO Box U1987, Perth, WA 6845, Australia.

C. J. Swain, 20 Bedwell Crescent, Booragoon, WA 6154, Australia. (c_swain@wt.com.au) 